Article

\title{
Improvement and Validation of Ranging Accuracy with YG-13A
}

\author{
Mingjun Deng ${ }^{1}$, Guo Zhang ${ }^{2, *}$, Ruishan Zhao ${ }^{3}$, Jiansong Li ${ }^{1}$, Shaoning Li ${ }^{2}$ \\ 1 School of Remote Sensing and Information Engineering, Wuhan University, Wuhan 430079, China; \\ dmj2008@whu.edu.cn (M.D.); jiansongli@whu.edu.cn (J.L.) \\ 2 State Key Laboratory of Information Engineering in Surveying, Mapping and Remote Sensing, Wuhan \\ University, Wuhan 430079, China; shaoningli@whu.edu.cn \\ 3 Liaoning Technical University, Fuxin 123000, China; zhaoruishan333@163.com \\ * Correspondence: guozhang@whu.edu.cn; Tel.: +86-139-0718-2592
}

\begin{abstract}
YG-13A represents the highest level of Chinese SAR satellites to date. In this paper, we report on experiments conducted to improve and validate ranging accuracy with YG-13A. We analyze the error sources in the YG-13A ranging system, such as atmospheric path delay, and transceiver channel delay. A real-time atmospheric delay correction model is established to calculate the atmospheric path delay, considering the troposphere delay and ionosphere delay. Six corner reflectors (CRs) were set up to ensure the accuracy of validation methods. Pixel location accuracies of up to $0.479-\mathrm{m}$ standard deviation can be achieved after a complete calibration. We further demonstrate that the adjustment of the CRs can cause a marginal loss of ranging precision. After eliminating this error, the ranging accuracy is improved to $0.237 \mathrm{~m}$. For YG-13A, a single frequency GPS receiver is used and the orbital nominal accuracy is $0.3 \mathrm{~m}$, which is the biggest factor restricting its ranging accuracy. Our results show that the ranging accuracy of YG-13A can achieve decimeter-level, which is lower than centimeter-level accuracy with TerraSAR-X loading a dual frequency GPS. YG-13A has great convenience in terms of access to control points and target location that does not depend on ground equipment.
\end{abstract}

Keywords: YG-13A; geometric accuracy; validation.

\section{Introduction}

The Synthetic Aperture Radar (SAR) is an effective microwave sensor with the capacity to work during the day and night under any weather conditions [1]. It can capture high resolution images of large areas, and thus plays an important role in Earth observations. In the past, SAR images were used merely for their 2-D imaging ability, while the inherently good geolocation accuracy of SAR systems was not adequately appreciated by the remote sensing community. For example, the pixel location accuracy requirements of Chinese SAR images were low for many years (in the order of $250 \mathrm{~m}$ ).

However, this appears to be changing. Since the 21st century, many SAR satellites have been launched into space from around the world. Their good geometric accuracy performances are owed to a large number of geometric calibration work [2]. In particular, TerraSAR-X pixel location accuracies of up to 2.6-cm standard deviation can be achieved after a single calibration, which is the best ranging accuracy reported for space-borne radar amplitude images thus far [3].

The Chinese YG-13A mission was launched in November 2015, equipped with a next-generation high-resolution SAR sensor in X-band, with a resolution of up to $0.5 \mathrm{~m}$. This means that China can now acquire high-resolution SAR images globally. YG-13A includes several improvements compared to previous Chinese SAR satellites, such as: (1) Higher image resolution due to a new sliding-spot imaging mode; (2) More flexible data acquisition due to the ability to image on both the left and right sides; (3) Improvement in the instruments' measuring accuracy.

With the increase of space-borne SAR image resolution, the influence of the ranging accuracy on the geometric and radiometric quality of the SAR image is becoming increasingly important [4]. 
In this paper, we focus on the ranging accuracy of YG-13A. We will show in the following sections that there are many error sources affecting the accuracy of slant range measurement. We will demonstrate using corner reflectors (CR) measurements, that the decimeter-level ranging accuracy of YG-13A can be achieved if we compensate for these errors using established models and available data. Although the ranging accuracy of YG-13A is lower than that of TerraSAR-X, it is in accordance with the theoretical precision.

\section{Range measurement accuracy of A SAR}

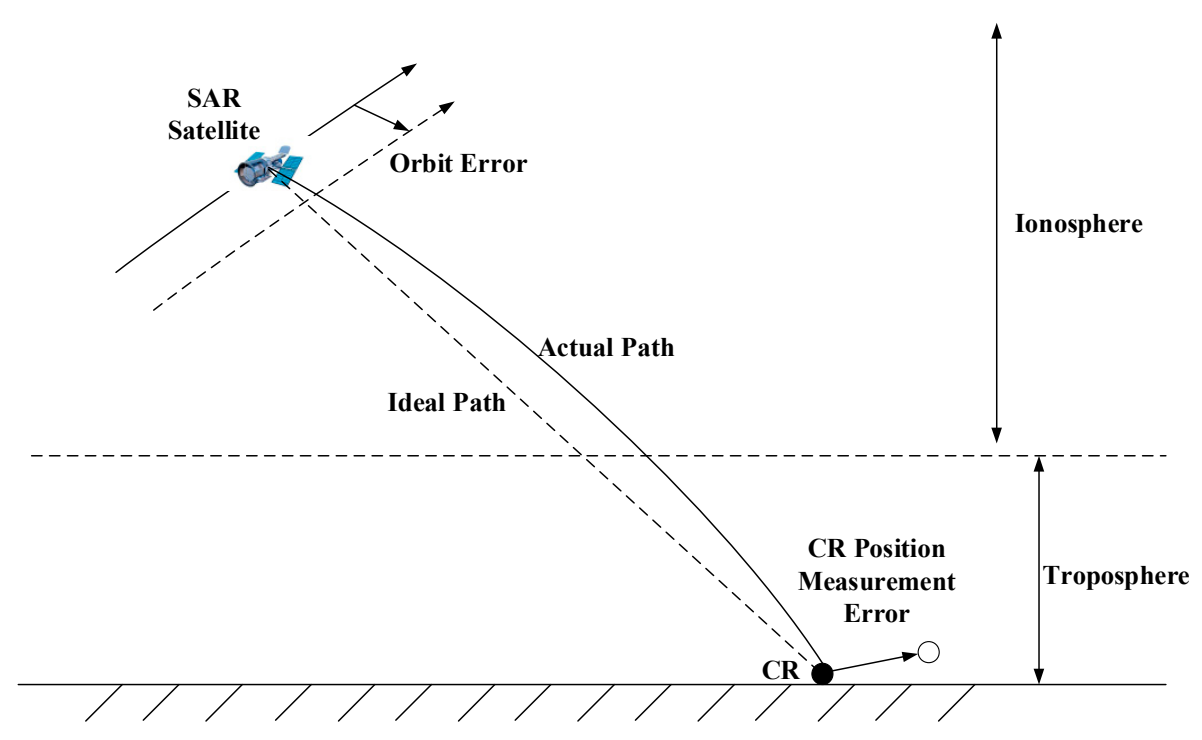

Figure 1. SAR imaging geometry

The SAR imaging geometry is represented in Figure 1, the distance $r$ between the target on the ground and the antenna phase center can be calculated from its range pixel position in the image by

$$
\mathrm{r}=r_{0}+\frac{c}{2 * f_{s}} * i+\Delta r
$$

where $r_{0}$ is the near range of the first range gate which is determined by the radar pulse propagation time; $i$ is the range pixel coordinate of the target in the image; $c$ is the propagation velocity of microwaves in the atmosphere; $f_{s}$ is the sampling frequency of the pulse; $\Delta r$ accounts for near range measurement error, and is usually determined by measuring the image location of corner reflectors positioned at a known distance $r$. Then, as can be seen in Figure 1 and equation (1), many error sources affect the accuracy of slant range measurement. In the following sections, the error sources of YG-13A ranging accuracy are described in detail.

\subsection{Orbit Accuracy}

This When the near range measurement error $\Delta r$ is determined by using a $\mathrm{CR}$, the position of the satellite is required to calculate the distance $r$. The accuracy of satellite orbit position affects the accuracy of the calculated range. By installing the GPS receiver on the satellite, the position of the GPS receiver is measured and converted to the position of the SAR antenna phase center.

For YG-13A, a single frequency GPS receiver is used. Single frequency GPS is advantageous in that it saves costs, reduces power consumption and reduces the pressure of data transmission. However, it cannot eliminate the influence of the ionospheric delay error through the dual frequency combination observation value, so its orbit precision is lower than the dual frequency GPS. After using an accurate orbit determination, a 3-D accuracy of $0.3 \mathrm{~m}$ for YG-13A is achieved. In contrast, a dual frequency GPS receiver is used for TerraSAR-X [5]. Therefore, a 3-D accuracy of $4.2 \mathrm{~cm}$, which is higher than that of YG-13A, is achieved

\subsection{Propagation Errors}


When the radar system is working, the radar signals travel through the medium between the antenna and ground. Because the refractive index of the atmosphere is not uniform, the radar signal encounters group delay in the ionosphere and troposphere (the so called Atmospheric Path Delay or APD) [6, 7]. Due to the existence of the atmospheric path delay, SAR slant range has several meters of measurement error. For SAR image signal, the atmospheric path delay $\Delta L$ is given by:

$\Delta L=\Delta L_{z} \cdot m(\theta)$

where $\Delta L_{z}$ is the zenith delay, $m(\theta)$ is the mapping functions [8].

There are many mapping function models, such as: Saastamoinen mapping function, Marini mapping function, and the Chao mapping function. These methods are highly accurate, but have complicated calculations. Taking into account the magnitude of atmospheric path delay and the required accuracy, the mapping function $1 / \cos \theta$ is used in $Y G-13 A$ processing [9-10]. This approach proves to be sufficient for our current analysis.

The zenith delay $\Delta L_{z}$ consists of two parts: ionosphere zenith delay $\Delta_{\text {iono }}$ and troposphere zenith delay $\Delta_{\text {trop }}$. For the troposphere, temperature, atmospheric pressure, and humidity are the basic parameters used to characterize the troposphere. At the same time, these three parameters are the main factors that affect the troposphere group delay [11]. The troposphere group delay caused by dry and wet air can be modeled well if the altitude, pressure, and water vapor content are known [12-14]. Troposphere zenith delay $\Delta_{\text {trop }}$ is given by

$$
\left\{\begin{array}{c}
\Delta_{\text {trop }}=\int_{z}^{\infty}(n(z)-1) d z \\
(n(z)-1)=10^{-6} N \\
N=k_{1}(\lambda) \frac{P_{d}}{T} z_{d}{ }^{-1}+k_{2}(\lambda) \frac{P_{w}}{T} z_{w}{ }^{-1}
\end{array}\right.
$$

where $n(z)$ is the refractive index along the zenith direction, $k_{1}(\lambda) \frac{P_{d}}{T} z_{d}{ }^{-1}$ is the delay caused by dry air, which is composed of oxygen and nitrogen, $k_{2}(\lambda) \frac{P_{w}}{T} z_{w}{ }^{-1}$ is the delay caused by wet air, which is composed of water vapor and $\mathrm{CO}_{2}, P_{d}$ is the pressure of dry air, $T$ is temperature, and $z_{d}$ is the compressibility of dry air. $P_{w}$ is the pressure of wet air, $z_{d}$ is the compressibility of wet air. $k_{1}(\lambda)$ and $k_{2}(\lambda)$ are related to the wavelength of the radar signal. The empirical equation proposed by Owens for $k_{1}(\lambda)$ and $k_{2}(\lambda)$ are listed as follows:

$$
\left\{\begin{array}{l}
k_{1}(\lambda)=0.237134+68.39397 \frac{130+\lambda^{2}}{\left(130-\lambda^{-2}\right)^{2}}+0.45473 \frac{38.9+\lambda^{-2}}{\left(38.9-\lambda^{-2}\right)^{2}} \\
k_{2}(\lambda)=0.648731+0.0174174 \lambda^{-2}+3.5575 \lambda^{-4}+6.1957 \lambda^{-6}
\end{array}\right.
$$

About $90 \%$ of the total troposphere zenith delay $\Delta_{\text {trop }}$ is caused by the dry air, which amounts to about $2.3 \mathrm{~m}$ at sea level, and the remaining $10 \%$ is caused by the wet air. In-timing atmospheric data from National Centers for Environmental Prediction (NCEP) are introduced in order to obtain pressure and temperature in SAR imaging time, and then ensure the accuracy of troposphere group delay correction [15].

In addition to troposphere zenith delay $\Delta_{\text {trop }}$, the ionosphere zenith delay $\Delta_{\text {iono }}$ is also considered. The velocity of the radar signal passing through the ionosphere is affected by the dispersion of the ionosphere [16, 17]. The delay in meters is given by

$$
\Delta_{\text {iono }}=\frac{40.28}{f^{2}} T E C
$$

here $f$ denotes the carrier frequency. TEC denotes vertical total electron content, which is often denoted in units of $10^{16}$ (called TECU). Figure 2 shows changes in TEC extracted for 24 hours a day over Songshan test fields. The TEC values are 1.5-30.3 TECU, the maximum TECU value appeared at noon, while the minimum value in the middle of the night, a zenith delay of $0.648 \mathrm{~cm}$ and 13.090 $\mathrm{cm}$ is caused by a value of $1.5 \mathrm{TECU}$ and $30.3 \mathrm{TECU}$. It is worth pointing out that since YG-13A is a low orbit satellite, it will experience only a part of the total column of electrons. Taking into account the YG-13A orbit accuracy of $0.3 \mathrm{~m}$, the difference of the ionosphere zenith delay value caused by the approximate calculation of the ionospheric electron content can be ignored. 


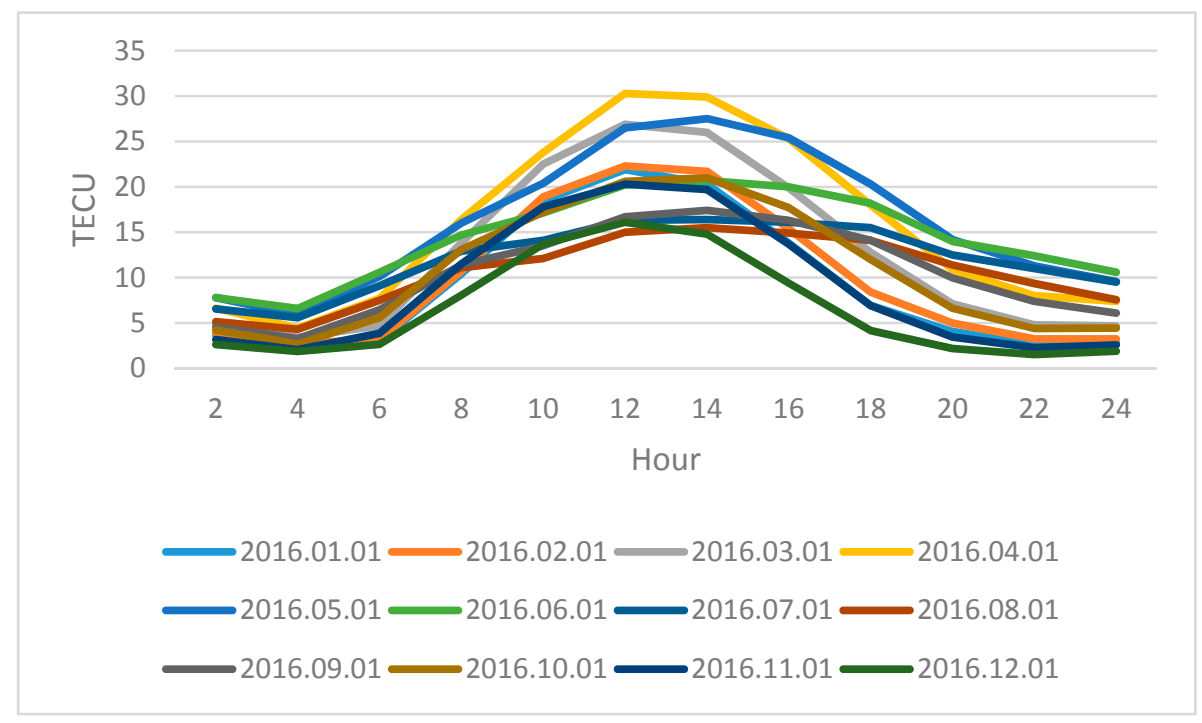

Figure 2. Ionospheric vertical TECU over Songshan Test fields

\subsection{Corner Reflector Location Measurement and Pixel Coordinate Extraction}

Calibration of SAR images because their reflecting area center can be determined accurately. Figure 3 shows the appearance of YG-13A CR and the performance of CR on a YG-13A sliding-spot image. As in the picture, the CR appears as bright point targets in SAR images. The rotation center coordinate of the CR was accurately measured with a GPS receiver and can be converted into the position of its vertex. Figure 4 shows the diagram of the coordinate calculation of the $C R$ vertex. The local coordinate system $\mathrm{O}-\mathrm{XYZ}$ is established. The $\mathrm{CR}$ vertex $\mathrm{P}$ coordinate can be calculated when the rotation center $\mathrm{O}$ coordinate and azimuth angle $\omega$ and elevation angle $\phi$ are known.

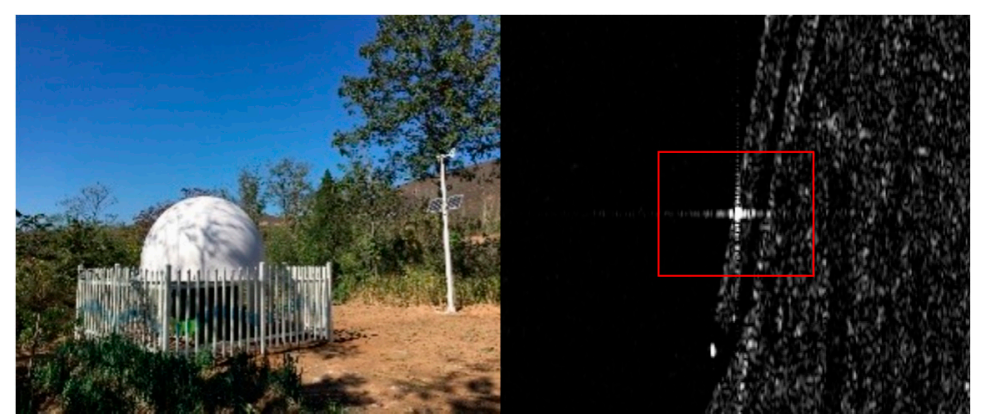

Figure 3. The appearance of CRs and the performance of CRs on SAR images

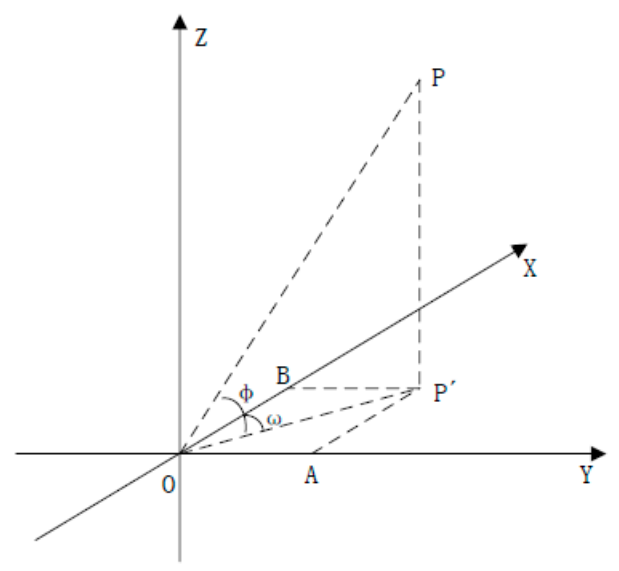

Figure 4. Diagram of the coordinate calculation of the $C R$ vertex 
The pixel coordinate of the CR vertex must be determined as well. The center line of the CR should coincide with the incident direction of the radar wave during radar running in order to reach the maximum radar cross-section. At this point, the brightest position on the image represents the pixel coordinate of the CR vertex. However, because the SAR image is a two-dimensional discrete signal, the peak of the CR pulse response does not necessarily correspond to the existing pixel signal on the SAR image. Interpolation calculation is necessary. Although there are many interpolation methods, the Sinc interpolation method can meet the requirements of the sub-pixel precision. Therefore, it is applied to YG-13A image processing.

\subsection{Transceiver Channel Delay, Sample Delay}

Except for propagation errors, there is also electronic delay of the instrument. It consists of transmitting channel delay and receiving channel delay. The transceiver channel delay $\mathrm{T}_{t r}$ of YG-13A in nanoseconds is given by

$$
\mathrm{T}_{t r}=t_{1}+2 \times \frac{1300 \sin \left(\theta_{\mathrm{R}}\right)+1700 \sin \left(\theta_{\mathrm{A}}\right)}{c}
$$

where $t_{1}$ is the delay time obtained by internal calibration, $2 \times \frac{1300 \sin \left(\theta_{R}\right)+1700 \sin \left(\theta_{A}\right)}{C_{0}}$ is related to radar range and azimuth beam scanning angle when the radar is working, $\theta_{R}$ and $\theta_{A}$ are the range and azimuth beam scanning angle, respectively, $\mathrm{c}$ is the propagation velocity of microwaves in the atmosphere. The value of YG-13A $\mathrm{T}_{t r}$ ranges from $300 \mathrm{~ns}$ to $400 \mathrm{~ns}$ and is obtained by ground test in-orbit tests.

There is also a sampling delay in the YG-13A ranging system due to the actual pulsewidth of the radar system being inconsistent with the nominal pulsewidth. In order to ensure that the radar signal is not disturbed by the noise, the actual pulsewidth is larger than the nominal pulsewidth. As show in Figure 5, both ends of the pulse carry useless information. The radar system takes the front edge of the pulse as the transmitting time, and the determination of the receiving time is related to the pulse peak position and the pulsewidth. Therefore, the range error caused by the inconsistency between the actual pulsewidth and the nominal pulsewidth is given by

$\mathrm{R}_{s_{-} d}=c *\left(\tau_{\text {act }}-\tau_{\text {nom }}\right) / 4$

where $\tau_{a c t}$ is the actual pulsewidth, $\tau_{\text {nom }}$ is the nominal pulsewidth, and $\mathrm{c}$ is the propagation velocity of microwaves in the atmosphere.

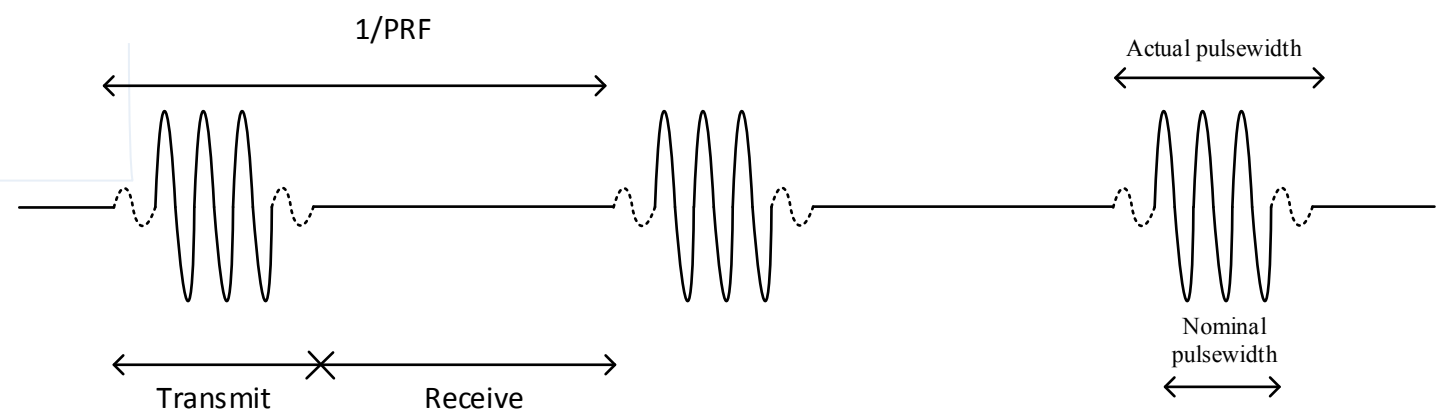

Figure 5. Radar pulse transmitting and receiving

\section{Experiment and Analysis}

\subsection{Experiment Data and Explanation}

Table 1 show the swath of each YG-13A imaging mode. As show in Figure 6, In order to cover all corner reflectors with a scene image, we set up six CRs in the range of $10 \times 10 \mathrm{~km}$ in the Songshan test field $\left(112.811^{\circ} \mathrm{E}-113.356^{\circ} \mathrm{E}, 34.255^{\circ} \mathrm{N}-34.634^{\circ} \mathrm{N}\right)$. We measure their positions with a GPS; the positioning accuracy in plane and elevation is within $0.1 \mathrm{~m}$. Songshan test field is located in Henan province, China, and the altitude of the region is between 100-1500 m. The test field is located in the central part of China, which includes a wide range of landforms, including plains, hills, and mountains. It is easy to obtain high quality SAR images. 
Table 1. Swath of YG-13A

\begin{tabular}{|c|c|c|}
\hline Imaging Mode & Resolution(m) & Swath $(\mathbf{K m})$ \\
\hline Sliding-Spot I & 0.5 & $10 \times 10$ \\
\hline Sliding-Spot II & 1 & $12 \times 12$ \\
\hline Strip-Map & 1.5 & Range: 12 \\
\hline
\end{tabular}

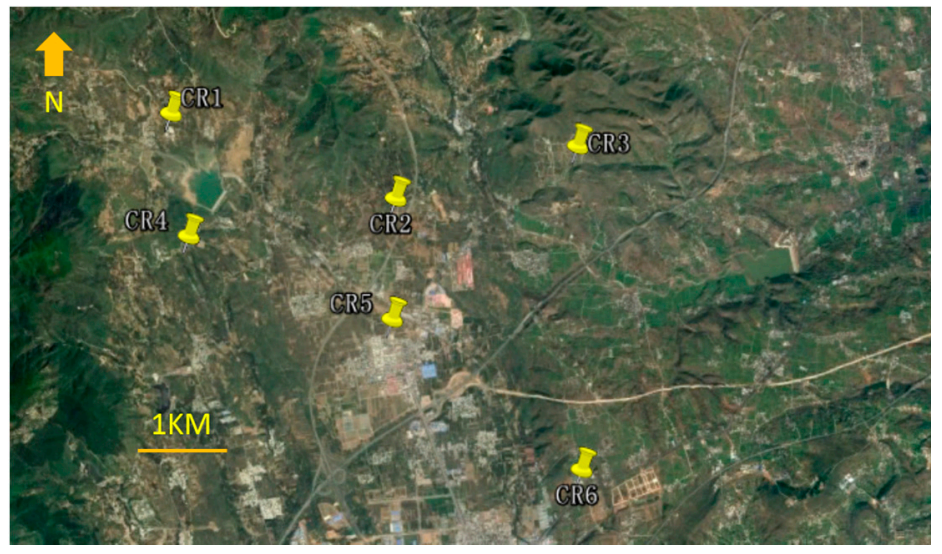

Figure 6. Songshan test field with six CRs $(\Delta)$

We acquire a series of 14 YG-13A stripmap images over the test field between December, 2015 and March, 2016. Table 2 shows the information of the image for experiment. Every time the radar is in function, and the satellite orbit types and incident angle are not the same, the orientation of the CRs must be adjusted according to the satellite parameters. In each of the 14 images, we measured the peak position of the CRs on the image using 64 pixels X 64 pixels windows centered at the CR, and performed a Sinc interpolation around the maximum. With this method, the previously determined theoretical accuracy can be achieved in practice.

Table 2. Information of YG-13A Images for experiment

\begin{tabular}{|c|c|c|c|c|c|c|}
\hline Date & OrbitType & LookSide & $\begin{array}{c}\text { Incidence } \\
\text { Angle }\left(^{\circ}\right)\end{array}$ & $\begin{array}{c}\text { Nominal } \\
\text { Pulsewidth } \\
\text { (us) }\end{array}$ & $\begin{array}{c}\text { Actual } \\
\text { Pulsewidth } \\
\text { (us) }\end{array}$ & $\begin{array}{c}\text { Band } \\
\text { Width } \\
(\mathrm{MHz})\end{array}$ \\
\hline 20151228 & Ascending & Right & 36.133 & 24.4 & 24.64 & 200 \\
\hline 20151229 & Descending & Right & 46.087 & 24.4 & 24.64 & 150 \\
\hline 20160103 & Descending & Right & 43.101 & 24.4 & 24.64 & 200 \\
\hline 20160107 & Descending & Right & 54.556 & 24.4 & 24.64 & 150 \\
\hline 20160116 & Ascending & Right & 26.849 & 24.4 & 24.64 & 200 \\
\hline $20160117 \mathrm{a}$ & Descending & Right & 50.896 & 24.4 & 24.64 & 150 \\
\hline $20160117 \mathrm{~b}$ & Ascending & Right & 48.872 & 24.4 & 24.64 & 150 \\
\hline 20160118 & Descending & Right & 31.364 & 24.4 & 24.64 & 200 \\
\hline 20160310 & Ascending & Right & 45.484 & 24.4 & 24.64 & 150 \\
\hline 20160311 & Descending & Right & 37.065 & 24.4 & 24.64 & 200 \\
\hline 20160315 & Ascending & Right & 47.996 & 24.4 & 24.64 & 150 \\
\hline 20160326 & Descending & Right & 23.586 & 24.4 & 24.64 & 200 \\
\hline 20160329 & Ascending & Right & 38.009 & 24.4 & 24.64 & 200 \\
\hline 20160330 & Ascending & Right & 53.777 & 24.4 & 24.64 & 150 \\
\hline
\end{tabular}

$a, b$ indicate that two images are acquired on the same day. 


\subsection{Result of Experiment and Analysis of Performance}

The range-Doppler model is commonly used for the rigorous positioning of SAR satellites [18]. The image pixel coordinates and the target ground position can be correlated very accurately by the three equations: distance equation, Doppler equation, and earth model equation. The pixel coordinate of CRs is then subtracted from the expected ideal geometric peak position (without considering the estimated propagation effects annotated in the product), which we called measured pixel coordinate. In addition, in the case of known geometric position of CRs, we can calculate the pixel coordinate of CRs by range-Doppler model, which we called calculated pixel coordinate. The difference between the calculated and measured can be converted to range error, which contains all the unknown errors and the known delays caused by the atmosphere. The results of range errors are shown in Table 3.

The additional (one-way) correction due to the atmospheric path delay is calculated by taking into account the zenith delay, the incidence angle, and the altitude of the CRs. The difference of the atmospheric path delay calculated by using each $C R$ in a scene is within $1 \mathrm{~cm}$, therefore, the atmospheric path delay of the whole scene is calculated by averaging the results. Figure 7 shows the atmospheric path delay of the 14 images of the experiment, with the increase of the incidence angle, the atmospheric path delay value becomes larger.

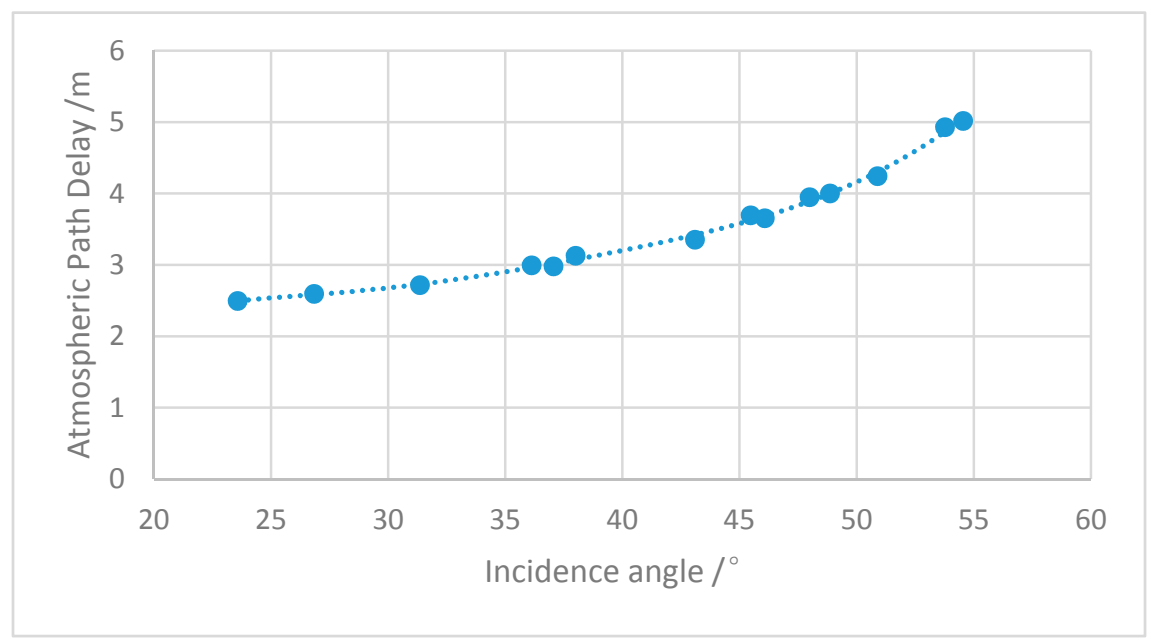

Figure 7. Relationship between atmospheric path delay and incident angle of 14 images

It's worth pointing out that transceiver channel delay represents range correction (two-ways). The stability of the transceiver channel delay and sample delay are apparent in Table 3 . With the different signal bandwidth, transceiver channel delay have a slightly difference. This is because the ranging precision of radar system is related to the sampling frequency, which is determined by signal bandwidth. Sample delay is $17.987 \mathrm{~m}$ according to equation (7).

As can be seen from Table 3, taking full account of multiple error factors, the ranging accuracy of YG-13A is $0.479 \mathrm{~m}$, while the average is close to zero. Taking account of a $0.3-\mathrm{m}$ orbital error, the residual range accuracy, which we achieved after those corrections, can still be improved. There is still room left for other error sources. Causes of result are analyzed as follows.

The test images contain two bandwidths: $200 \mathrm{MHz}$ and $150 \mathrm{MHz}$. Table 4 and Table 5 show the statistics for the standard deviation of the $200 \mathrm{MHz}$ and $150 \mathrm{MHz}$ images, respectively. The ranging accuracy is not improved, indicating that the error caused by the difference of the imaging bandwidth is not significant. Table 6 and Table 7, respectively, show the statistics for the standard deviation of two orbital type images. The result indicate that the ranging accuracy is improved from $0.479 \mathrm{~m}$ to $0.239 \mathrm{~m}$ and $0.237 \mathrm{~m}$. The residual range accuracy is in accordance with what we expect from orbital error estimates. Because the orientation of the CRs needs to be adjusted when the orbital type of the images are not the same, the error is introduced. The achieved accuracy is even better than the orbital accuracy of $0.3 \mathrm{~m}$, this indicates that the orbital accuracy may be better than $0.3 \mathrm{~m}$. 
Table 3. Absolute localization residuals of CRs

\begin{tabular}{|c|c|c|c|c|c|}
\hline Date & $\begin{array}{c}\text { Range error } \\
{[\mathrm{m}]}\end{array}$ & $\begin{array}{c}\text { Atmosphere } \\
\text { Path Delay } \\
{[\mathrm{m}]}\end{array}$ & $\begin{array}{c}\text { Transceiver } \\
\text { Channel } \\
\text { Delay }[\mathrm{m}]\end{array}$ & $\begin{array}{c}\text { Sample } \\
\text { Delay [m] }\end{array}$ & $\begin{array}{c}\text { Residual } \\
\text { CRs } \\
{[\mathrm{m}]}\end{array}$ \\
\hline 20151228 & 76.530 & 2.992 & 112.718 & 17.987 & -0.808 \\
\hline 20151229 & 77.855 & 3.654 & 111.127 & 17.987 & 0.651 \\
\hline 20160103 & 78.188 & 3.351 & 112.718 & 17.987 & 0.491 \\
\hline 20160107 & 78.568 & 5.017 & 111.127 & 17.987 & 0.001 \\
\hline 20160116 & 76.588 & 2.592 & 112.718 & 17.987 & -0.350 \\
\hline $20160117 \mathrm{a}$ & 78.079 & 4.242 & 111.127 & 17.987 & 0.287 \\
\hline $20160117 \mathrm{~b}$ & 77.259 & 4.000 & 111.127 & 17.987 & -0.291 \\
\hline 20160118 & 77.706 & 2.715 & 112.718 & 17.987 & 0.645 \\
\hline 20160310 & 76.538 & 3.693 & 111.127 & 17.987 & -0.705 \\
\hline 20160311 & 77.933 & 2.977 & 112.718 & 17.987 & 0.610 \\
\hline 20160315 & 77.077 & 3.946 & 111.127 & 17.987 & -0.419 \\
\hline 20160326 & 77.021 & 2.494 & 112.718 & 17.987 & 0.181 \\
\hline 20160329 & 77.425 & 3.127 & 112.718 & 17.987 & -0.048 \\
\hline 20160330 & 78.150 & 4.927 & 111.127 & 17.987 & -0.327 \\
\hline Stdev & 0.649 & 0.779 & 0.795 & 0 & 0.479 \\
\hline mean & 77.494 & 3.551 & 111.922 & 17.987 & -0.0058 \\
\hline
\end{tabular}

Table 4. Result of $200 \mathrm{MHz}$ images

\begin{tabular}{|c|c|c|c|c|c|c|c|}
\hline Date & 20151228 & 20160103 & 20160116 & 20160118 & 20160311 & 20160326 & 20160329 \\
\hline $\begin{array}{c}\text { Residual } \\
\text { CRs [m] }\end{array}$ & -0.808 & 0.491 & -0.350 & 0.645 & 0.610 & 0.181 & -0.048 \\
\hline Stdev & \multicolumn{7}{|c|}{$\mathbf{0 . 5 0 2}$} \\
\hline mean & \multicolumn{7}{|c|}{$\mathbf{0 . 1 0 3}$} \\
\hline
\end{tabular}

Table 5. Result of $150 \mathrm{MHz}$ images

\begin{tabular}{|c|c|c|c|c|c|c|c|}
\hline Date & 20151229 & 20160107 & $20160117 \mathrm{a}$ & $20160117 \mathrm{~b}$ & 20160310 & 20160315 & 20160330 \\
\hline $\begin{array}{c}\text { Residual } \\
\text { CRs[m] }\end{array}$ & 0.651 & 0.001 & 0.287 & -0.291 & -0.705 & -0.419 & -0.327 \\
\hline Stdev & \multicolumn{7}{|c|}{$\mathbf{0 . 4 2 7}$} \\
\hline mean & \multicolumn{7}{|c|}{$\mathbf{- 0 . 1 1 4}$} \\
\hline
\end{tabular}

Table 6. Result of ascending-orbit and right-lookside images

\begin{tabular}{|c|c|c|c|c|c|c|c|}
\hline Date & 20151228 & 20160116 & $20160117 \mathrm{~b}$ & 20160310 & 20160315 & 20160329 & 20160330 \\
\hline $\begin{array}{c}\text { Residual } \\
\text { CRs[m] }\end{array}$ & -0.808 & -0.350 & -0.291 & -0.705 & -0.419 & -0.048 & -0.327 \\
\hline Stdev & \multicolumn{7}{|c|}{$\mathbf{0 . 2 3 9}$} \\
\hline mean & \multicolumn{7}{|c|}{$\mathbf{0 . 4 2 1}$} \\
\hline
\end{tabular}

Table 7. Result of descending-orbit and right-lookside images

\begin{tabular}{|c|c|c|c|c|c|c|c|}
\hline Date & 20151229 & 20160103 & 20160107 & $20160117 \mathrm{a}$ & 20160118 & 20160311 & 20160326 \\
\hline $\begin{array}{c}\text { Residual } \\
\text { CRs[m] }\end{array}$ & 0.651 & 0.491 & 0.001 & 0.287 & 0.645 & 0.610 & 0.181 \\
\hline Stdev & \multicolumn{7}{|c|}{$\mathbf{0 . 2 3 7}$} \\
\hline mean & \multicolumn{7}{|c|}{$\mathbf{0 . 4 0 9}$} \\
\hline
\end{tabular}




\section{Conclusions}

In this study, the error sources in the YG-13A ranging system were analyzed. The ranging accuracy of YG-13A reached $0.237 \mathrm{~m}$ after the atmospheric path delay correction, and the transceiver channel delay correction. Considering the $0.3 \mathrm{~m}$ orbital nominal accuracy, this result was close to the theoretical limit level. The ranging accuracy of YG-13A is mainly limited by the orbit accuracy determined by a single frequency GPS. The result of this study proved that the range measurement of a SAR does not use the attitude parameter, and can achieve high precision.

The atmospheric delay correction method using external data was selected to calculate the atmospheric delay correction. For X-band satellites, the ionospheric delay correction is very small, and atmospheric path delay is mainly influenced by tropospheric delay. The ionospheric delays can be considered to improve the results marginally. We ignored some of the dynamics of the ionosphere. This is acceptable for the X-band but certainly not for the C-and L-bands, and it is need to be considered in the centimeter-level ranging accuracy validation.

Because the test images have two kinds of orbit-type: ascending and descending, it is necessary to adjust the CRs, which introduce some errors. In the centimeter-level ranging accuracy validation experiment, we can set up some fixed CRs and acquire a series of images as repeat-pass acquisitions on the same orbit and incidence angle. Decimeter-level ranging accuracy can satisfy the requirement of high precision control points and target positioning. There is still room for improvement at the centimeter-level precision. With centimeter-level ranging accuracy, absolute measurements of volcanoes or glaciers are possible without the use of ground equipment and without the use of SAR interferometry.

Acknowledgments: The authors would like to thank the editors and anonymous reviewers for their constructive suggestions.

Author Contributions: Mingjun Deng wrote the paper and conducted the experiments. Guo Zhang guided the experiments and the structure of the paper. Ruishan Zhao and Jiansong Li checked the paper and gave some suggestions. Atmospheric data is provided by Shaoning Li.

Conflicts of Interest: The authors declare no conflict of interest.

\section{References}

1. Gumming I G and Wong F H. Synthetic aperture radar imaging algorithm and implementation $[\mathrm{M}]$. Electronic Industries Press, Beijing, 2007: 1-3.

2. Josef M., Marwan Y., Robert M. TerraSAR-X System Performance Characterization and Verification [J]. IEEE Transactions on geoscience and remote sensing, 2010, 48, 660-676..

3. Michael E., Christian M., Peter S. Imaging Geodesy-Toward Centimeter-Level Ranging Accuracy With TerraSAR-X [J]. IEEE Transactions on geoscience and remote sensing, 2011, 49, 661-671.

4. Liu X., Liu J., Hong W. The Analysis of the Precision in Space-borne SAR Image Location [J]. Journal of remote sensing, 2006, 01, 76-81.

5. Yoon Y., Michael E., Montenbruck O. "TerraSAR-X precise trajectory estimation and quality assessment," IEEE Transactions on geoscience and remote sensing., 2009, 47, 1859-1868.

6. Adrian S., Michael J., David S. Influence of Atmospheric Path Delay on the Absolute Geolocation Accuracy of TerraSAR-X High-Resolution Products [J]. IEEE Transactions on geoscience and remote sensing, 2010, 48, 751-758.

7. Jehle M., Perler D., Small D. Estimation of Atmospheric Path Delays in TerraSAR-X Data using Models vs. Measurements [J]. Sensors. 2008, 8, 8479-8491.

8. Li S., Xiao J., Ma Y., Zhou H., Guo X. Study on Atmospheric Refraction Delay Correction for Satellite Laser Altimeter System [J]. Optics \& Optoelectronic technology, 2013, 01, 7-11.

9. Wang C., Zhang H. Comparison of Atmospheric Refraction Delay Mapping Function [J]. Geospatial Information. 2009, 6, 85-87.

10. Zhu T., Zhu J., Zhang X. Atmospheric Refraction Numerical Fitting Research Based on Mapping Function and Neural Network [J]. Journal of geodesy and geoinformation science. 2007, 36, 290-295.

11. Doin M., Lasserre C., Peltzer G. Corrections of Stratified Tropospheric Delays in SAR Interferometry: Validation with Global Atmospheric Models [J]. Journal of Applied Geophysics. 2009, 69, 35-50.

12. Davis J., Herring T., Shapiro I. Geodesy by Radio Interferometry: Effects of Atmospheric Modeling Errors on Estimates of Baseline Length [J]. Radio Science. 1985, 20(6), 1593-1607. 
13. Marini J. Correction of satellite tracking data for an arbitrary troposphere profile [J]. Radio Science, 1972, $7(2,223-231$.

14. Chao C. Troposphere calibration model for mariner Mars 1971 [R]. Technical Report 32-1587, JPL, Pasadena, California, 1974, 61-76.

15. Chen Q., Song S., Zhu W. An Analysis of the Accuracy of Zenith Tropospheric Delay Calculated from ECMWF/NCEP Data over Asian Area [J]. Chinese Journal of Geophysics. 2012, 55(5), 1541-1548.

16. Herring T., Quinn K. Atmospheric Delay Correction to GLAS Laser Altimeter Ranges. Available online: http://www.csr.utexas.edu/glas/atbd.html, 2001

17. Zhang H., Ping J., Zhu W., Huang Cheng. Brief review of the ionospheric delay model [J]. Progress in astronomy, 2006, (01), 16-26.

18. Fei W. Research and Application of the RPC model Geometry Processing for Space-borne SAR and Space-borne InSAR. [D]. Wuhan, Wuhan University, 2012. 